\title{
Anything that can go wrong, will go wrong: should a pre-existing epidural catheter be discarded for an intrapartum cesarean section?
}

\author{
Duk Kyung Kim \\ Department of Anesthesiology and Pain Medicine, Samsung Medical Center, Sungkyunkwan University School of \\ Medicine, Seoul, Korea
}

As more parturients demand pain-free labor, epidural labor analgesia (ELA) is becoming widespread due to its well-defined benefits over other systemic medications [1]. When a parturient who has received ELA requires a cesarean section (CS), it is generally accepted that she should be anesthetized via the epidural catheter placed during labor. However, the failure rate for such conversion of ELA to epidural surgical anesthesia (ESA) is $1.7-38 \%$, varying depending on the definition of failure [2].

When failure of extension from ELA to ESA occurs, administration of general anesthesia or repeat neuraxial blockade such as epidural or spinal anesthesia. However, this unanticipated course of action can represent a serious threat to patient safety due to the hazards associated with managing an obstetric airway, performing a spinal technique in the presence of a partial epidural blockade, or titration of appropriate analgesic and sedative medications, as well as reduced patient satisfaction $[3,4]$.

Yoon et al. [5], in the present issue of the Korean Journal of Anesthesiology, carefully described the efficacy of spinal anesthesia without attempting to extend the epidural for intrapartum CS anesthesia. In their prospective, non-blinded, parallel-arm, randomized trial, they compared the rate of pain-free surgery between ESA and spinal anesthesia following ELA for intrapartum CS. As a result, the failure rate of achieving pain-free sur-

Corresponding author: Duk Kyung Kim, M.D., Ph.D.

Department of Anesthesiology and Pain Medicine, Samsung Medical Center, Sungkyunkwan University School of Medicine, 81, Irwon-ro, Gangnam-gu, Seoul 06351, Korea

Tel: 82-2-3410-0343, Fax: 82-2-3410-6626

Email: dikei@hanmail.net

ORCID: https://orcid.org/0000-0002-6555-2100

Korean J Anesthesiol 2017 August 70(4): 373-374

https://doi.org/10.4097/kjae.2017.70.4.373 gery was significantly higher in the ESA group than in the spinal anesthesia group ( $15.3 \%$ vs. $2.5 \%, \mathrm{P}<0.001)$, although there was no statistical difference between the two groups in the rate of conversion to general anesthesia $(2.5 \%$ [4/163] in the ESA group vs. $1.3 \%[2 / 160]$ in the spinal anesthesia group, $\mathrm{P}=0.697)$.

Such findings are not surprising considering the high failure rate for the conversion from ELA to ESA for intrapartum CS. In addition, spinal anesthesia essentially produces a denser sensory and motor blockade and does not involve the sacral sparing phenomenon to the same degree as for epidural anesthesia.

Nevertheless, the major finding of this study was that the rate of failed conversion from ELA to ESA for intrapartum CS remained high at $15.3 \%$, even after meticulous confirmation of a functional epidural. Because they studied parturients of urgency category 3 (i.e., needing early delivery but without maternal or fetal compromise) by the Royal College of Anaesthetists, urgency for surgical readiness was not reflected in the high failure rate in their study. One of the major reasons for not utilizing ELA in some studies was having an emergency CS [3]. This strategy may have originated from the perception that it takes longer to convert from ELA to ESA than to induce general anesthesia or spinal anesthesia.

The authors suggested possible mechanisms underlying failed conversion of ELA to ESA, including more severe intraoperative anxiety in parturients undergoing intrapartum CS versus those undergoing elective CS under regional anesthesia and the decreased threshold of pain perception during intrapartum CS due to central sensitization.

Considering the unacceptably high failure rate of extending ELA to ESA, proactive strategies should be implemented by anesthesiologists performing intrapartum CS. The first strategy is to identify the risk factors for failed conversion and prepare for the possible hazards associated with an at-risk epidural catheter.

(c) This is an open-access article distributed under the terms of the Creative Commons Attribution Non-Commercial License (http://creativecommons.org/ licenses/by-nc/4.0/), which permits unrestricted non-commercial use, distribution, and reproduction in any medium, provided the original work is properly cited. 
A second could be preemptive adoption of general anesthesia or spinal anesthesia without attempting to extend the epidural.

Which strategy is better for improving safety and quality? Unfortunately, the present study by Yoon et al. [5] does not yield a clear answer to that question. Their study was not sufficiently powered to evaluate the safety profile (complications related to high block, respiratory compromise associated with supplemental analgesic and sedative medications, or maternal and fetal complications related to surgical delay or difficulty). It was only powered to evaluate a single indicator of efficacy (rate of painfree surgery).

Unanticipated changes in anesthesia during intrapartum CS can lead to serious complications. Therefore, clinicians should be aware of the higher risk associated with insufficient epidural anesthesia via a pre-existing epidural catheter (even if it functioned well during labor analgesia) and weigh the remote risk of insufficient block against the risk of complications resulting from emergent general anesthesia or spinal anesthesia.

\section{References}

1. Hawkins JL. Epidural analgesia for labor and delivery. N Engl J Med 2010; 362: 1503-10.

2. Lee S, Lew E, Lim Y, Sia AT. Failure of augmentation of labor epidural analgesia for intrapartum cesarean delivery: a retrospective review. Anesth Analg 2009; 108: 252-4.

3. Bauer ME, Kountanis JA, Tsen LC, Greenfield ML, Mhyre JM. Risk factors for failed conversion of labor epidural analgesia to cesarean delivery anesthesia: a systematic review and meta-analysis of observational trials. Int J Obstet Anesth 2012; 21: 294-309.

4. Hawkins JL, Koonin LM, Palmer SK, Gibbs CP. Anesthesia-related deaths during obstetric delivery in the United States, 1979-1990. Anesthesiology 1997; 86: 277-84.

5. Yoon HJ, Do SH, Yun YJ. Comparing epidural surgical anesthesia and spinal anesthesia following epidural labor analgesia for intrapartum cesarean section: a prospective randomized controlled trial. Korean J Anesthesiol 2017; 70: 412-9. 